\title{
Article
}

\section{ILC for Non-Linear Hyperbolic Partial Difference Systems}

\author{
Meryem Hamidaoui (1) and Cheng Shao *(1) \\ Institute of Advanced Control Technology, Dalian University of Technology, Dalian 116024, China; \\ meryem@mail.dlut.edu.cn \\ * Correspondence: cshao@dlut.edu.cn
}

check for

updates

Citation: Hamidaoui, M.; Shao, C. ILC for Non-Linear Hyperbolic Partial Difference Systems. Mathematics 2021, 9, 3076. https:// doi.org/10.3390/math9233076

Academic Editors: Alberto Ferrero and Ravi P. Agarwal

Received: 13 October 2021

Accepted: 26 November 2021

Published: 29 November 2021

Publisher's Note: MDPI stays neutral with regard to jurisdictional claims in published maps and institutional affiliations.

Copyright: (c) 2021 by the authors. Licensee MDPI, Basel, Switzerland. This article is an open access article distributed under the terms and conditions of the Creative Commons Attribution (CC BY) license (https:// creativecommons.org/licenses/by/ $4.0 /)$.

\begin{abstract}
This paper discusses the iterative learning control problem for a class of non-linear partial difference system hyperbolic types. The proposed algorithm is the PD-type iterative learning control algorithm with initial state learning. Initially, we introduced the hyperbolic system and the control law used. Subsequently, we presented some dilemmas. Then, sufficient conditions for monotone convergence of the tracking error are established under the convenient assumption. Furthermore, we give a detailed convergence analysis based on previously given lemmas and the discrete Gronwall's inequality for the system. Finally, we illustrate the effectiveness of the method using a numerical example.
\end{abstract}

Keywords: PD-type iterative learning control; non-linear partial difference systems; hyperbolic partial difference systems; initial learning state; convergence

\section{Introduction}

The partial difference system (PDS) is a type of DPS. It originates from the discretization in time and space of the partial differential systems. Its state variables have two or more independent variables. Therefore, the research of PDE and PDS has an enormous application background. There are many sources with regard to partial difference equations/systems both in theory and application [1-9].

For example, in [4], the authors built a partial difference system model for researching image processing. For the problem of the molecular orbital model, Reference [5] applies the partial difference system. The authors in [3] used the partial difference system to study the solution of partial differential equations. In [6], for two linear partial difference equations with constant coefficients, the authors presented solution stability conditions.

However, not many works consider the control issue for PDS. Owing to the addition of the number of variables in the system state, like the control problem of PDE [10,11], the control designs for the partial differential system are more intricate than ODEs.

This is unfortunate because of the mathematical complexity resulting from the partial differential equation forms. In such an approach, great efficiency control habitually requires that the DPS be approximated with plenty of ODEs, thus, dramatically enhancing the complexity of the calculations. The computational cost linked with these lumping strategies can be limiting for some processes and might not improve the performance that explains the additional computational cost. Current control techniques are necessary to surmount the computational cost.

As a branch of intelligent control, the iterative learning control was proposed by Arimoto in 1984. It is a comparatively recent addition to the control engineering toolkit, as per [12]. Usually, new capacities and skills are developed by trials and corrections. Thus, the ILC is founded on the acquisition of knowledge in practice, which is the learning mechanism generally used by human beings. It was proved to be a decent method to handle the systems with repetitiveness. The ILC has become an alternative method to solve the control problem, since it is simple, quick, efficient, and has perfect tracking performance. ILC has been used to track a settled target in many systems, such as fractional 
systems [13], ordinary difference systems [14,15], and distributed parameter systems or partial differential systems [16,17]. However, there are a few subjects on ILC for partial difference systems $[18,19]$, where the authors consider the parabolic PDS.

The PD-type ILC is a preferred process to improve the convergence rate and achieve robustness against uncertainty. Many papers discuss the use of the PD-ILC method. For example, a PD-ILC algorithm was presented in [20] to find the optimal path of robots. In [21], the PD-ILC was proposed to a class of switched discrete systems with external noises and uncertainties. An open-closed loop PD-ILC with variable gains was proposed in [22] for a class of time-varying nonlinear systems.

Here we study the ILC problem of hyperbolic PDEs. The PD-type ILC is proposed for the system. By using a discrete form inequality/formula, the convergence conditions are given. It is shown that choosing the learning gain parameter through the iterative learning process can assure the convergence of the output tracking error between the actual output and the given desired output.

Compared with the existing literature, the principal characteristics of this work are summarized below.

1. Because the research object is a class of hyperbolic partial difference systems, the PDtype ILC updating law is proposed. The convergence conditions of the error are given in the iteration domain.

2. It is known that the ILC method is based on some assumptions, one of which is the fixed and equal nature of the initial conditions. Here, the initial state also has an iterative updating law, which means the initial conditions are unfixed and no longer equal.

3. In this work, A new form of the output function is proposed to obtain a monotone and faster convergence.

4. The solution of this class of equations does not have a general form, so to prove the convergence of the proposed method, we use some mathematical tools, such as the Young inequality and the Gronwall inequality.

The structural components of this paper are arranged as follows. In Section 2, we introduce the systems, and the ILC scheme is described. Analysis of convergence is shown in Section 3. In section 4, an example is proposed to illustrate the effectiveness of the proposed method. Finally, Section 5 summarizes this paper.

Notation: For a function $y(i, j) \in R$, take the norm: $\|y(., j)\|_{L^{2}}=\sqrt{\sum_{a}^{b} y(i, j)^{2}}$, and the $\lambda$-norm of the function is given by: $\|y\|_{\lambda}=\sup _{j \in[0, j]}\left(\lambda^{j} y(., j) \|_{L^{2}}^{2}\right.$.

\section{Problem Statement}

Various industrial processes are DPS, in which states vary in both space and time, such as sheet coating processes, polymer-extrusion, and fixed bed reactors. Mathematical representations of such systems habitually achieved using conservation laws are a type of PDE. When the processes have space variation, the high-accuracy PDE forms in modelbased control for DPSs are used, increasing the constraint handling performance and the monitoring.

Hyperbolic PDEs portray the dynamics of diverse industrial processes implicated in convection with minor diffusion effects (e.g., fiber splines, plug flow reactors, and fluid heat exchangers). The continuous non-linear hyperbolic system is given as

$$
\begin{cases}\frac{\partial^{2} y}{\partial \mu^{2}}(s, \mu)-A \Delta y(s, \mu)=F(y(s, \mu))+B u(s, \mu) ; & (s, \mu) \in Q ; \\ z(s, \mu)=C y(s, \mu)+D \int_{0}^{\mu} u(s, \tau) d \tau & (s, \mu) \in \bar{Q}\end{cases}
$$

Here, $A, B, C$, and $D$ are positive real constants. $\Delta y(s, \mu)=\frac{\partial^{2} y}{\partial s^{2}}(s, \mu) . \Omega=(a, b)$, $a, b \in R, Q=\Omega \times(0, T]$, and $\bar{Q}=\bar{\Omega} \times[0, T]$, The function $\mathrm{F}$ is continuous non-linear 
function supposed to satisfy the Lipschitz condition (at least locally), i.e., $\| F\left(y_{1}(s, \mu)\right)-$ $F\left(y_{2}(s, \mu)\right)\left\|\leq f_{0}\right\| y_{1}(s, \mu)-y_{2}(s, \mu) \|$.

Contrary to parabolic PDEs, the hyperbolic differential spatial operator includes eigenvalues of the almost equal or the same amount of energy. As a result, the handling with hyperbolic PDEs necessitates an analysis of the nature of the systems in infinitedimensional. It is very complicated, but transferring the problem into PDS makes it easy and uncomplicated.

Hence, the partial difference system is given by

$$
\left\{\begin{array}{l}
\Delta_{1}^{2} y(i, j-1)-A \Delta_{2}^{2} y(i-1, j)=F(y(i, j))+B u(i, j) \\
z(i, j)=C y(i, j)+D \sum_{\tau=0}^{j} u(i, \tau)
\end{array}\right.
$$

where $i \in\{1,2, \ldots, I\}, j \in\{0,1, \ldots, J\}$ denote the space and time variables respectively and I, J are given integers. $y(i, j), z(i, j), u(i, j) \in R$ are the system state, output, and input, respectively. Where the non-linearity term still satisfies Lipschitz condition

The partial differences symbols $\Delta$ are defined as

$$
\left\{\begin{array}{l}
\Delta_{1} y(p, l)=y(p, l+1)-y(p, l) \\
\Delta_{1}^{2} y(p, l-1)^{2}=y(p, l+1)-2 y(p, l)+y(p, l-1) \\
\Delta_{2} y(p, l)=y(p+1, l)-y(p, l) \\
\Delta_{2}^{2} y(p-1, l)=y(p+1, l)-2 y(p, l)+y(p-1, l)
\end{array}\right.
$$

where $p \in\{1,2, \ldots, I\}, l \in\{0,1, \ldots, J\}$.

In this paper, for the systems modeled by the nonlinear hyperbolic type PDS, an ILC scheme is presented it can effectively drive the process output to the required one. For these hyperbolic systems, our proposed method provides monotonic convergence of the system with the desired output. We give the convergence theorem and its proof using some basic lemmas. Finally, we introduce a numerical simulation to show the efficiency of the method (see Figure 1) and a comparison with some ILC schemes.

The learning system as the $k$ th iteration is given as follows:

$$
\left\{\begin{array}{l}
\Delta_{1}^{2} y_{k}(i, j-1)-A \Delta_{2}^{2} y_{k}(i-1, j)=F\left(y_{k}(i, j)\right)+B u_{k}(i, j) \\
z_{k}(i, j)=C y_{k}(i, j)+D \sum_{\tau=0}^{j} u_{k}(i, \tau)
\end{array}\right.
$$

The considered controller in this paper is as follows:

$$
\left\{\begin{array}{l}
u_{k+1}(i, j)=u_{k}(i, j)+L_{1} e_{k}(i, j)+L_{2} \Delta_{1} e_{k}(i, j-1) \\
y_{k+1}(i, 0)=y_{k}(i, 0)+L_{3} e_{k}(i, 0) \\
y_{k+1}(i, 1)=y_{k}(i, 1)+L_{4} e_{k}(i, 1)
\end{array}\right.
$$

$k=1,2, \ldots$ represent the iteration numbers. $L_{1}, L_{2}, L_{3}$ and $L_{4}$ are positive define matrix. $e_{k}$ is the tracking error and it is defined as follows, $e_{k}(i, j)=z_{d}(i, j)-z_{k}(i, j)$, where $z_{d}(i, j)$ is the desired function (output function), $z_{k}(i, j)$ is the output at the $k$ th iteration, respectively. 


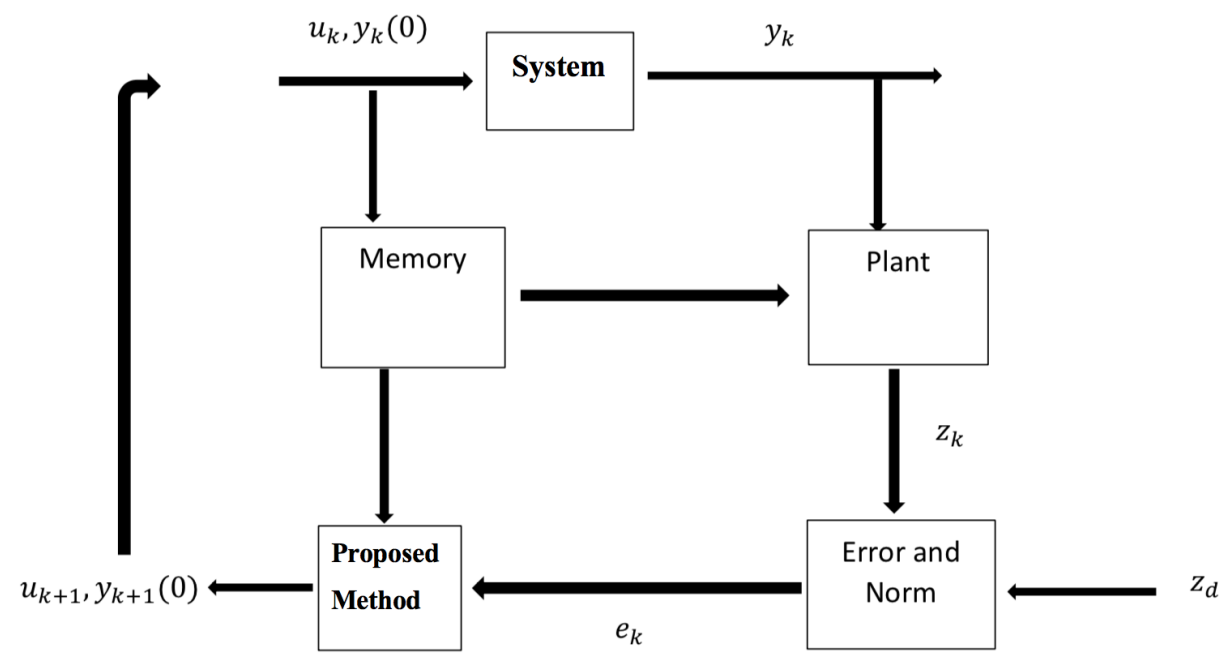

Figure 1. The block diagram of proposed method.

Assumption 1. For the system (4), we assume that there is a unique system input $u_{d}(i, j) \in R$ satisfying that

$$
\left\{\begin{array}{l}
\Delta_{1}^{2} y_{d}(m, l-1)-A \Delta_{2}^{2} y_{d}(m-1, l)=F\left(y_{d}(m, l)\right)+B u_{d}(m, l) \\
z_{d}(m, l)=C y_{d}(m, l)+D \sum_{\tau=0}^{l} u_{d}(m, \tau)
\end{array}\right.
$$

Remark 1. Assumption 1 signifies that for the needed trajectory, there exists a realizable pair of inputs and states such that the system (4) can reach the wanted output.

Remark 2. The boundary value conditions of the system (4) are assumed to be the same for all iterations and to be equal to the desired output boundary value $y_{k}(0, j)=y_{d}(0, j), y_{k}(I+1, j)=$ $y_{d}(I+1, j), 0 \leq j \leq J$.

\section{Convergence Analysis}

Lemma 1. Suppose that $a_{k}$ and $b_{k}$ are real non-negative sequence realizing, $a_{k+1} \leq \rho a_{k}+b_{k}$. If $0 \leq \rho<1$ and $b_{k} \rightarrow 0$ when $k \rightarrow \infty$, then $a_{k} \rightarrow 0$ when $k \rightarrow \infty$.

The proof is easy to complete.

Lemma 2 (Discrete Gronwall inequality). Let the constant sequences $y(i), c(i)$ and $d(i)$ be real sequences which satisfy $y(i+1) \leq c(i) y(i)+d(i), 0 \leq c(i), 0 \leq i$. Then, we have

$$
y(j) \leq \prod_{i=0}^{j-1} c(i) y(0)+\sum_{i=1}^{j-1} d(i) \prod_{s=i+1}^{j-1} c(s)
$$

For more detail refer to Xie and Cheng, 1996.

Lemma 3. For $h(k, l) \in R$ real function, there exist two positive constant $H_{1}$ and $H_{2}$ such that

$$
\|h\|_{\lambda} \leq H_{1}\left\|\Delta_{1} h\right\|_{\lambda}+H_{2}\|h(i, 0)\|_{L^{2}}^{2}
$$


Proof of Lemma 3. From (3) we have,

$$
\begin{aligned}
\Delta_{1} h(m, l) & =h(m, l+1)-h(m, l) \\
h(m, l+1) & =\Delta_{1} h(m, l)+h(m, l) \\
\sum_{m=1}^{I} h(m, l+1)^{2} & \leq 2 \sum_{m=1}^{I} \Delta_{1} h(m, l)^{2}+2 \sum_{m=1}^{I} h(m, l)^{2}
\end{aligned}
$$

where $m \in\{1,2, \ldots, I\}, l \in\{0,1, \ldots, J\}$.

Applying Lemma 2, we get

$$
\begin{gathered}
\|h(., l+1)\|_{L^{2}}^{2} \leq 2\|h(., 0)\|_{L^{2}}^{2}+2 \sum_{s=1}^{l-1}\left\|\Delta_{1} h(., s)\right\|_{L^{2}}^{2} \\
\|h(., l+1)\|_{L^{2}}^{2} \leq 2\|h(., 0)\|_{L^{2}}^{2}+2\left\|\Delta_{1} h\right\|_{\lambda} \sum_{s=1}^{l-1} \lambda^{-s} \\
\|h\|_{\lambda} \leq 2\|h(., 0)\|_{L^{2}}^{2}+\frac{2 \lambda^{J+1}}{\lambda-1}\left\|\Delta_{1} h\right\|_{\lambda}
\end{gathered}
$$

This end the proof, with $H_{1}=\frac{2 \lambda^{J+1}}{\lambda-1}$ and $H_{2}=2$.

Theorem 1. Suppose that Assumption 1 is satisfied. If

$$
\max \left(\left(1-C L_{3}-D L_{1}\right)^{2},\left(1-C L_{4}-D\left(L_{1}-L_{2}\right)\right)^{2}\right) \leq \sigma<1
$$

and

$$
\rho=4\left(1-D L_{2}\right)^{2} \leq \epsilon<\frac{1}{4}
$$

under the effect of the control law (5), then the iterative process of the system (4) is convergent, i.e. $\left\|e_{k}\right\|_{\lambda} \rightarrow 0$ when $k \rightarrow \infty$

Proof. The proof of the theorem is divided to three steps. Let $w_{k}=y_{k}-y_{k+1}, v_{k}=$ $u_{k}-u_{k+1}$, and $F_{k}(i, j)=F\left(y_{k}(i, j)\right)$

1. Firstly, we proof that $\left\|e_{k}(., 0)\right\|_{L^{2}} \rightarrow 0$ when $k \rightarrow \infty$

$$
\begin{aligned}
e_{k+1}(i, 0) & =z_{d}(i, 0)-z_{k+1}(i, 0)=e_{k}(i, 0)+z_{k}(i, 0)-z_{k+1}(i, 0) \\
& =e_{k}(i, 0)-C L_{3} e_{k}(i, 0)-D L_{1} e_{k}(i, 0)=\left(1-C L_{3}-D L_{1}\right) e_{k}(i, 0) \\
\left(e_{k+1}(i, 0)\right)^{2} & \leq\left(1-C L_{3}-D L_{1}\right)^{2} e_{k}(i, 0)^{2} \\
\sum_{i=1}^{I}\left(e_{k+1}(i, 0)\right)^{2} & \leq\left(1-C L_{3}-D L_{1}\right)^{2} \sum_{i=1}^{I} e_{k}(i, 0)^{2}
\end{aligned}
$$

According to the theorem and to Lemma $1,\left\|e_{k}(., 0)\right\|_{L^{2}} \rightarrow 0$ when $k \rightarrow \infty$.

2. Secondly, we proof that $\left\|e_{k}(., 1)\right\|_{L^{2}} \rightarrow 0$ when $k \rightarrow \infty$

$$
\begin{aligned}
e_{k+1}(i, 1) & =z_{d}(i, 1)-z_{k+1}(i, 1)=e_{k}(i, 1)+z_{k}(i, 1)-z_{k+1}(i, 1) \\
& =e_{k}(i, 1)-C L_{4} e_{k}(i, 1)-D L_{1} e_{k}(i, 0)-D L_{1} e_{k}(i, 1)-D L_{2} e_{k}(i, 1)+D L_{2} e_{k}(i, 0) \\
& =\left(1-C L_{4}-D\left(L_{1}+L_{2}\right)\right) e_{k}(i, 1)+D\left(L_{1}+L_{2}\right) e_{k}(i, 0) \\
\left(e_{k+1}(i, 1)\right)^{2} & \leq\left(1-C L_{4}-D\left(L_{1}+L_{2}\right)\right)^{2} e_{k}(i, 1)^{2}+D^{2}\left(L_{1}+L_{2}\right)^{2} e_{k}(i, 0)^{2} \\
\sum_{i=1}^{I}\left(e_{k+1}(i, 1)\right)^{2} & \leq\left(1-C L_{4}-D\left(L_{1}+L_{2}\right)\right)^{2} \sum_{i=1}^{I} e_{k}(i, 1)^{2}+D^{2}\left(L_{1}+L_{2}\right)^{2} \sum_{i=1}^{I} e_{k}(i, 0)^{2}
\end{aligned}
$$

According to Theorem 1, Lemma 1, and because $\left\|e_{k}(., 0)\right\|_{L^{2}} \rightarrow 0$ when $k \rightarrow \infty$ we get $\left\|e_{k}(., 1)\right\|_{L^{2}} \rightarrow 0$ when $k \rightarrow \infty$ 
3. Finally, to proof the convergence, we will proof that $\left\|\Delta_{1} e_{k}\right\|_{\lambda} \rightarrow 0$ when $k \rightarrow \infty$, then use Lemma 3 to proof that $\left\|e_{k} \mid\right\|_{\lambda} \rightarrow 0$ when $k \rightarrow \infty$.

$$
\begin{gathered}
\Delta_{1} e_{k+1}(i, j)=\Delta_{1}\left(z_{d}(i, j)-z_{k+1}(i, j)\right)=\Delta_{1}\left(e_{k}(i, j)+z_{k}(i, j)-z_{k+1}(i, j)\right) \\
=\Delta_{1} e_{k}(i, j)+C \Delta_{1} w_{k}(i, j)+D \Delta_{1} \sum_{l=0}^{j} v_{k}(i, l) \\
=\left(1-D L_{2}\right) \Delta_{1} e_{k}(i, j)+C \Delta_{1} w_{k}(i, j)+D L_{1} e_{k}(i, 0)-D L_{1} e_{k}(i, j+1) \\
\left(\Delta_{1} e_{k+1}(i, j)\right)^{2} \leq 4\left(1-D L_{2}\right)^{2}\left(\Delta_{1} e_{k}(i, j)\right)^{2}+4\left(D L_{1} e_{k}(i, j+1)\right)^{2} \\
+4\left(C \Delta_{1} w_{k}(i, j)\right)^{2}+4\left(D L_{1} e_{k}(i, 0)\right)^{2} \\
=4\left(1-D L_{2}\right)^{2}\left(\Delta_{1} e_{k}(i, j)\right)^{2}+4 D^{2} L_{1}^{2}\left(e_{k}(i, j+1)\right)^{2} \\
+4 C^{2}\left(\Delta_{1} w_{k}(i, j)\right)^{2}+4 D^{2} L_{1}^{2}\left(e_{k}(i, 0)\right)^{2} \\
\text { Let } \rho=4\left(1-D L_{2}\right)^{2}, \quad \\
\left\|\Delta_{1} e_{k+1}(., j)\right\|_{L^{2}}^{2} \leq \rho\left\|\Delta_{1} e_{k}(., j)\right\|_{L^{2}}^{2}+4 D^{2} L_{1}^{2}\left\|e_{k}(., j+1)\right\|_{L^{2}}^{2} \\
\quad+4 C^{2}\left\|\Delta_{1} w_{k}(., j)\right\|_{L^{2}}^{2}+4 D^{2} L_{1}^{2}\left\|e_{k}(., 0)\right\|_{L^{2}}^{2} \\
\quad \\
\lambda^{j}\left\|\Delta_{1} e_{k+1}(., j)\right\|_{L^{2}}^{2} \leq \lambda^{j} \rho\left\|\Delta_{1} e_{k}(., j)\right\|_{L^{2}}^{2}+4 \lambda^{j} D^{2} L_{1}^{2}\left\|e_{k}(., j+1)\right\|_{L^{2}}^{2} \\
+4 \lambda^{j} C^{2}\left\|\Delta_{1} w_{k}(., j)\right\|_{L^{2}}^{2}+4 \lambda D^{j} L_{1}^{2}\left\|e_{k}(., 0)\right\|_{L^{2}}^{2} \\
\quad \\
\quad 4 D^{2} L_{1}^{2} \lambda\left\|e_{k}\right\|\left\|_{\lambda}+4 \lambda^{J} D^{2} L_{1}^{2}\right\| e_{k}(., 0) \|_{L^{2}}^{2}
\end{gathered}
$$

Using Lemma 3, we get

$$
\begin{aligned}
\left\|\Delta_{1} e_{k+1} \mid\right\|_{\lambda} & \leq \rho\left\|\Delta_{1} e_{k}||_{\lambda}+4 C^{2}\right\| \Delta_{1} w_{k}\left\|_{\lambda}+4 D^{2} L_{1}^{2} H_{1}\right\| \Delta_{1} e_{k} \|\left.\right|_{\lambda} \\
& +\left.4 D^{2} L_{1}^{2} H_{2}\left\|e_{k}(., 0)\right\|\right|_{L^{2}} ^{2}+4 \lambda^{J} D^{2} L_{1}^{2}\left\|e_{k}(., 0)\right\| \|_{L^{2}}^{2}
\end{aligned}
$$

To finish the proof we must estimate $\left\|\Delta_{1} w_{k}\right\|_{\lambda}$,

$$
\begin{aligned}
\Delta_{1} w_{k}(i, j)- & \Delta_{1} w_{k}(i, j-1)=A \Delta_{2}^{2} w_{k}(i-1, j)+F_{k}(i, j)-F_{k+1}(i, j)+B v_{k}(i, j) \\
\Delta_{1} w_{k}(i, j)^{2} & =\left(\Delta_{1} w_{k}(i, j-1)+A \Delta_{2}^{2} w_{k}(i-1, j)\right. \\
& \left.+F_{k}(i, j)-F_{k+1}(i, j)+B v_{k}(i, j)\right)\left(\Delta_{1} w_{k}(i, j-1)+A \Delta_{2}^{2} w_{k}(i-1, j)\right. \\
& \left.+F_{k}(i, j)-F_{k+1}(i, j)+B v_{k}(i, j)\right) \\
& \leq 4 \Delta_{1} w_{k}(i, j-1)^{2}+4 A \Delta_{2}^{2} w_{k}(i-1, j)^{2}+4 f_{0} w_{k}(i, j)^{2}+4 B v_{k}(i, j)^{2} \\
\sum_{i=1}^{I} \Delta_{1} w_{k}(i, j)^{2} \leq & \sum_{i=1}^{I}\left(4 \Delta_{1} w_{k}(i, j-1)^{2}+4 A \Delta_{2}^{2} w_{k}(i-1, j)^{2}\right. \\
& \left.+4 f_{0} w_{k}(i, j)^{2}+4 B v_{k}(i, j)^{2}\right)
\end{aligned}
$$

from another side we have,

$$
\sum_{i=1}^{I} \Delta_{2}^{2} w_{k}(i-1, j)^{2} \leq 18 \sum_{i=1}^{I} w_{k}(i, j)^{2}
$$


then

$$
\begin{aligned}
\left\|\Delta_{1} w_{k}(., j)\right\| \|_{L^{2}}^{2} & \leq 4\left\|\Delta_{1} w_{k}(., j-1)\right\|_{L^{2}}^{2}+4 f_{0}\left\|w_{k}(., j)\right\|_{L^{2}}^{2} \\
& +72 A\left\|w_{k}(., j)\right\|_{L^{2}}^{2}+4 B\left\|v_{k}(., j)\right\|_{L^{2}}^{2} \\
\left\|\Delta_{1} w_{k}(., j)\right\| \|_{L^{2}}^{2} & \leq 4\left\|\Delta_{1} w_{k}(., j-1)\right\|_{L^{2}}^{2} \\
& +\left(4 f_{0}+72 A\right)\left\|w_{k}(., j)\right\|_{L^{2}}^{2}+4 B\left\|v_{k}(., j)\right\|_{L^{2}}^{2}
\end{aligned}
$$

Using Lemma 2, we get

$$
\begin{aligned}
\left\|\Delta_{1} w_{k}(., j-1)\right\|_{L^{2}}^{2} & \leq \prod_{l=0}^{j-1} 4\left\|\Delta_{1} w_{k}(., 0)\right\|_{L^{2}}^{2} \\
& +\sum_{l=1}^{j-1}\left(\left(4 f_{0}+72 A\right)\left\|w_{k}(., l)\right\|_{L^{2}}^{2}+4 B\left\|v_{k}(., l)\right\|_{L^{2}}^{2}\right) \\
& \leq 4\left\|\Delta_{1} w_{k}(., 0)\right\|_{L^{2}}^{2}+\left(4 f_{0}+72 A\right) \sum_{l=1}^{j-1}\left\|w_{k}(., l)\right\|_{L^{2}}^{2} \\
& +4 B \sum_{l=1}^{j-1}\left\|v_{k}(., l)\right\|_{L^{2}}^{2} \\
\left\|\Delta_{1} w_{k}(., j-1)\right\|_{L^{2}}^{2} & \leq 4|| \Delta_{1} w_{k}(., 0)\left\|_{L^{2}}^{2}+\left(4 f_{0}+72 A\right)\right\| w_{k} \|{ }_{\lambda} \sum_{l=1}^{j-1} \lambda^{-l} \\
& +4 B\left\|v_{k}\right\| \sum_{\lambda}^{j-1} \sum_{l=1}^{-l} \lambda^{-l} \\
\left\|\Delta_{1} w_{k}\right\| \|_{\lambda} & \leq 4\left\|\Delta_{1} w_{k}(., 0)\right\|_{L^{2}}^{2}+\left(4 f_{0}+72 A\right) \frac{\lambda^{J}-1}{\lambda-1}\left\|w_{k}\right\|_{\lambda} \\
& +4 B \frac{\lambda^{J}-1}{\lambda-1}\left\|v_{k}\right\|_{\lambda}
\end{aligned}
$$

Using Lemma 3, we get

$$
\begin{aligned}
\left\|\Delta_{1} w_{k}\right\|_{\lambda} & \leq 4\left\|\Delta_{1} w_{k}(., 0)\right\|_{L^{2}}^{2}+\left(4 f_{0}+72 A\right) \frac{\lambda^{J}-1}{\lambda-1}\left(H_{1}\left\|\Delta_{1} w_{k}\right\|_{\lambda}\right. \\
& \left.+H_{2}\left\|w_{k}(., 0)\right\|_{L^{2}}^{2}\right)+4 B \frac{\lambda^{J}-1}{\lambda-1}\left\|v_{k}\right\|_{\lambda} \\
\left.\left\|\Delta_{1} w_{k}\right\|\right|_{\lambda} & \leq 4 \alpha_{\lambda}\left\|\Delta_{1} w_{k}(., 0)\right\|_{L^{2}}^{2}+\beta_{\lambda} \alpha_{\lambda}\left\|w_{k}(., 0)\right\|_{L^{2}}^{2}+4 B \gamma_{\lambda}\left\|v_{k}\right\|_{\lambda}
\end{aligned}
$$

with $\alpha_{\lambda}=1-\left(4 f_{0}+72 A\right) H_{1} \gamma_{\lambda}, \beta_{\lambda}=\left(4 f_{0}+72 A\right) H_{2} \gamma_{\lambda}$, and $\gamma_{\lambda}=\frac{\lambda^{J}-1}{\lambda-1}$

replacing (11) in (7) we get,

$$
\begin{aligned}
\left\|\Delta_{1} e_{k+1}\right\|_{\lambda} & \leq \rho\left\|\Delta_{1} e_{k}\right\|_{\lambda}+4 C^{2}\left(4 \alpha_{\lambda}\left\|\Delta_{1} w_{k}(., 0)\right\|_{L^{2}}^{2}\right) \\
& +4 C^{2}\left(\beta_{\lambda} \alpha_{\lambda}\left\|w_{k}(., 0)\right\|_{L^{2}}^{2}+4 B \gamma_{\lambda}\left\|v_{k}\right\|_{\lambda}\right) \\
& +4 D^{2} L_{1}^{2} H_{1}\left\|\Delta_{1} e_{k}\right\|_{\lambda}+4 D^{2} L_{1}^{2} H_{2}\left\|e_{k}(., 0)\right\|_{L^{2}}^{2}+4 \lambda^{J} D^{2} L_{1}^{2}\left\|e_{k}(., 0)\right\|_{L^{2}}^{2}
\end{aligned}
$$


or, $\left\|w_{k}(., 0)\right\|_{L^{2}}^{2}=L_{3}^{2}\left\|e_{k}(., 0)\right\|_{L^{2}}^{2}$

now, we estimate $\left\|v_{k}\right\|_{\lambda}$ and $\left\|\Delta_{1} w_{k}(., 0)\right\|_{L^{2}}^{2}$

$$
\begin{gathered}
\left\|v_{k}\right\|_{\lambda} \leq 2 L_{1}^{2}\left\|e_{k}\right\|_{\lambda}+2 L_{2}^{2}\left\|\Delta_{1} e_{k}\right\|_{\lambda} \\
\left\|\Delta_{1} w_{k}(., 0)\right\|_{L^{2}}^{2} \leq L_{3}^{2}\left\|e_{k}(., 0)\right\|_{L^{2}}^{2}+L_{4}^{2}\left\|e_{k}(., 1)\right\|_{L^{2}}^{2}
\end{gathered}
$$

then we get, for $\lambda$ small enough and using Theorem $1,\left\|\Delta_{1} e_{k}\right\|_{\lambda} \rightarrow 0$ when $k \rightarrow \infty$, then use Lemma 3, $\left\|e_{k}\right\|_{\lambda} \rightarrow 0$ when $k \rightarrow \infty$.

\section{Numerical Simulation}

For a numerical simulation we chose Sin-Gordon equation.

The Sin-Gordon equation describes many phenomena in physics and engineering, such as the mention of rigid pendular attached to a stretched wire, the propagation of fluxons in Josephson junctions, the dynamics of coupled pendulums, and the continuum limits of the crystalline lattices.

Without losing generation taking: $a=0, b=2 \pi$, many contributions were realized in the control of this equation, such as,

$$
\left\{\begin{array}{l}
\Delta_{1}^{2} y_{k}(p, l)-\Delta_{2}^{2} y_{k}(p, l)=u_{k}(p, l)+\sin \left(y_{k}(p, l)\right) \\
z_{k}(p, l)=C y_{k}(p, l)+D \sum_{\tau=0}^{l} u_{k}(p, \tau)
\end{array}\right.
$$

According to the theorem condition we took, $L_{1}=0.6, L_{2}=0.9, L_{3}=0.4, L_{4}=0.6$ and $\lambda=0.5$ with $z_{d}(p, l)=l \sin (p / 50)+\sin ((I+1-p) / 20) \cos (l / 50)$, the method converge after 7 iteration (Table 1). The simulation results of the proposed method are shown in Figures 2-5.

Figure 2 shows the $\lambda$-norm of the error-iteration number and the error-space-time. Figure 3 shows the $\lambda$-norm of the error-iteration number and the iteration for the proposed method, the PD-ILC, the P-ILC, and the D-ILC, where we can see that our proposed method is faster. Figure 4 shows the system output and the desired output in the 7 nd iteration when $l=0$ and $l=1$. Figure 5 presents the 7 th iteration of the system output, and the desired output.

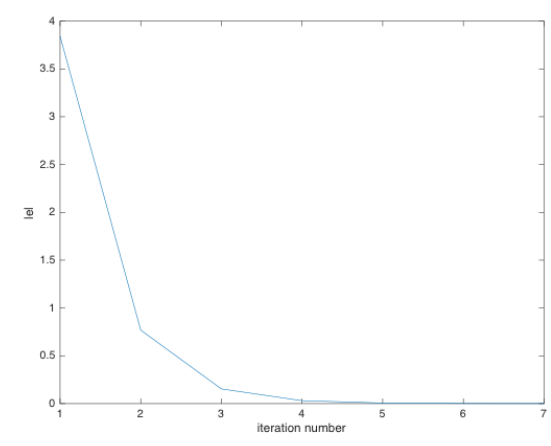

(a)

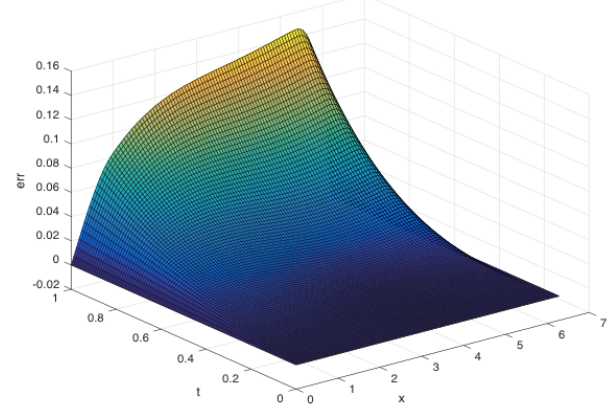

(b)

Figure 2. (a) The $\lambda$-norm of the error-iteration number and (b) the error-space-time. 


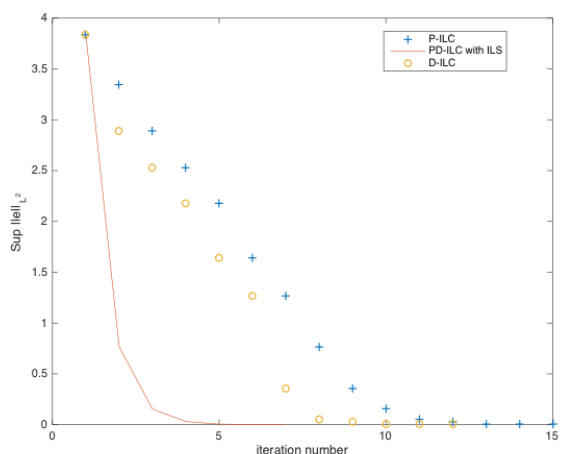

(a)

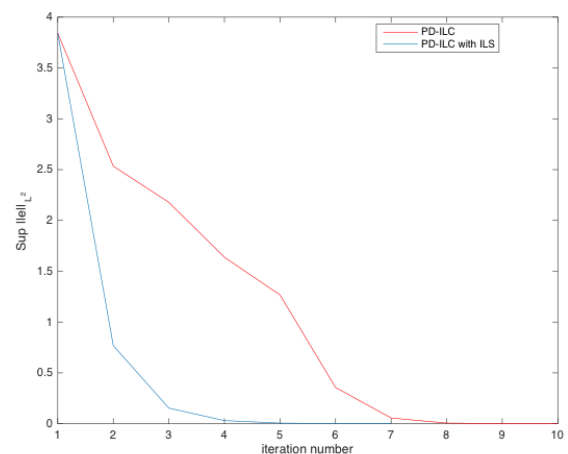

(b)

Figure 3. (a) The $\lambda$-norm of the error-iteration number of the proposed method and P-ILC and D-ILC, and $(\mathbf{b})$ the $\lambda$-norm of the error-iteration number of the proposed method and PD-ILC.

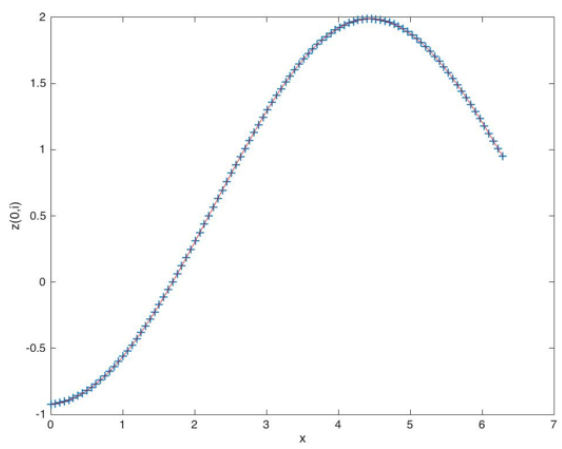

(a)

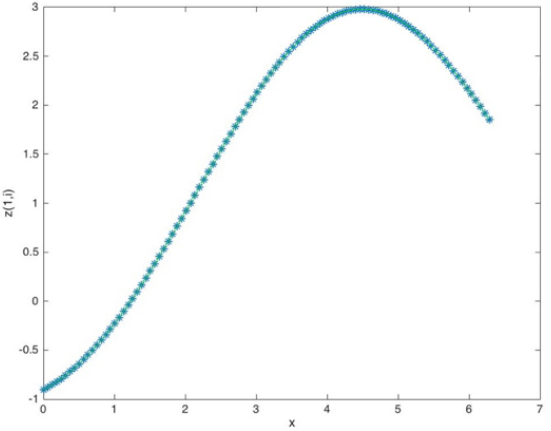

(b)

Figure 4. (a) The desired output and the system output at $k=7(l=0)$ and (b) the desired output and the system output at $k=7(l=1)$.

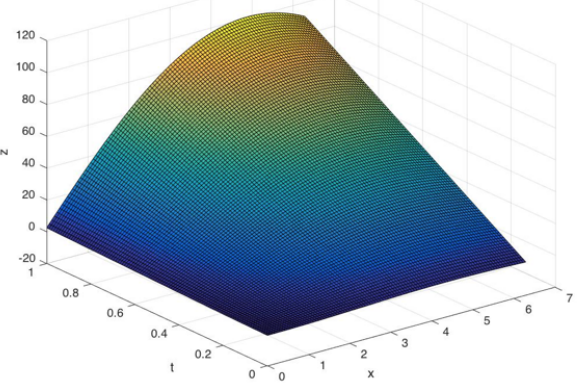

(a)

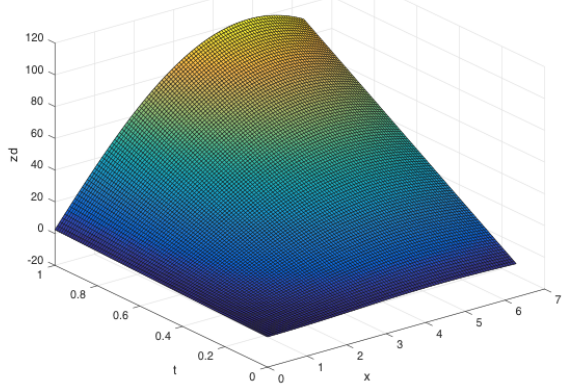

(b)

Figure 5. (a) The output at the 7th iteration and (b) the desired output.

Table 1. The norm value of the error and iteration number.

\begin{tabular}{cccccccc}
\hline Iteration Number & $\mathbf{1}$ & $\mathbf{2}$ & $\mathbf{3}$ & $\mathbf{4}$ & $\mathbf{5}$ & $\mathbf{6}$ & $\mathbf{7}$ \\
\hline error norm & 3.842 & 0.7684 & 0.1537 & 0.0307 & 0.0061 & 0.0012 & 0.0002 \\
\hline
\end{tabular}

\section{Discussion}

According to the previous analysis, the proposed scheme outperforms most of the ILC approaches in terms of second-order nonlinear PDS control in terms of performance and speed. This resounding success underlines the effectiveness of the PD controller, the law 
of iterative updating of initial states, and the new output function. It confirms that the proposed controller can achieve the desired result in fewer iterations with a monotonous convergence of the error to zero. These are the advantages of our proposed method.

The proposed method is theoretically proven and with a numerical experiment only. However, its results and its simplicity proves that the proposed scheme can handle realworld applications as well. This will be confirmed in our future work.

Remark 3. Generally, the ILC suffers from a transient error, which can cause problems in practice. Here, we proved the monotone convergence of the error for the presented ILC. The monotonic convergence is one of the proposed solutions to the problem of transient error. However, to be more assured in practice, the BEA [23] method is applied. This algorithm deals with this issue by tracking the magnitude of the error during the iteration. As soon as it reaches a pre-determined limit value, the current execution is terminated. The correction of the input signals before the next iteration is performed only until the previous one has been concluded. We didn't mention this method because we will consider the convergence and choice of the limit value in our future work.

\section{Conclusions}

In this paper, we introduced a new approach to the ILC. It is based on A PD-type ILC algorithm with ILS and a new output function. Also, we have discussed the distributed control of a fundamental class of equations, the nonlinear hyperbolic partial difference system. The main contribution of this work is that we have developed a new technique of control to handle this class of equations. Another contribution is that the convergence of the proposed method was given using the Gronwall Lemma and other mathematical tools instead of utilizing the solution forms.

Furthermore, we illustrated the effectiveness of this proposed control technique of simulation verification. Compared to all available methods in the literature, the simulation results indicate that the proposed method has better estimation performance, and states of estimation error systems converge to zero monotonous and faster with almost no transient error.

Author Contributions: M.H.: Conceptualization, Methodology, Software, Validation, Formal analysis, Writing-Original Draft preparation. C.S.: Validation, Writing—Review and Editing, Supervision, Formal analysis. All authors have read and agreed to the published version of the manuscript.

Funding: This work is partly financial supported by the High-tech Research and Development Program of China (2014AA041802).

Institutional Review Board Statement: Not applicable.

Informed Consent Statement: Not applicable.

Data Availability Statement: Not applicable.

Conflicts of Interest: The authors declare no conflict of interest.
Abbreviations
The following abbreviations are used in this manuscript:
DPS Distributed Parameter Systems
PDE Partial Differential Equation
ODE Ordinary Differential Equation
ILC Iterative Learning Control
ILS Initial Learning State
PD-type Propotional Derivative controller
BEA Bounded Error Algorithm 


\section{References}

1. Cheng, S.S. Advances in Discrete Mathematics and Applications Volume 3: Partial Difference Equations; Elaydi, S., Ladas, G., Eds.; Taylor \& Francis: London, UK, 2009.

2. Bridgman, T.; Hereman, W.; Quispel, G.R.; van der Kamp, P.H. Symbolic computation of lax pairs of partial difference equations using consistency around the cube. Found. Comput. Math. 2013, 13, 517-544. [CrossRef]

3. Morton, K.W.; Mayers, D.F. ANumerical Solution of Partial Differential Equations: An Introduction; Cambridge University Press: London, UK, 2005.

4. Bertrma, E.S.; Leo, O.C. Resistive grid image filtering: Input/output analysis via the CNN Framework. IEEE Trans. Circuits Syst.-I Fundam. Theory Appl. 1999, 39, 531-548.

5. Li, X.P. Molecular orbital method of partial difference equation. J. Chem. 1982, 40, 688-698.

6. Lin, Y.Z.; Cheng, S.S. Stability criteria for two partial difference equations. J. Comput. Appl. Math. 1996, 32, 87-103. [CrossRef]

7. Liu, S.T.; Cheng, S.S. Nonexistence of positive solutions of a nonlinear partial difference equation. J. Math. 1997, $28,51-58$. [CrossRef]

8. Ta, V.T.; Elmoataz, A.; Lézoray, O. Partial Difference Equations over Graphs: Morphological Processing of Arbitrary Discrete Data. In Computer Vision-ECCV 2008. Lecture Notes in Computer Science; Forsyth, D., Torr, P., Zisserman, A., Eds.; Springer: Berlin/Heidelberg, Germany, 2008; Volume 5304.

9. Xie, Z.; Li, H.B. Formal integrability criteria for nonlinear partial difference equations. Acta Appl. Math. 2009, $108,279-297$. [CrossRef]

10. Luo, B.; Wu, H.N. Online policy iteration algorithm for optimal control of linear hyperbolic PDE systems. J. Process Control 2012, 22, 1161-1170. [CrossRef]

11. Luo, B.; Wu, H.N. Approximate optimal control design for nonlinear one-dimensional parabolic PDE systems using empirical eigenfunctions and neural network. IEEE Trans. Syst. Man Cybern.-Part B 2012, 42, 1538-1549.

12. Arimoto, S.; Kawamura, S.; Miyazaki, F. Bettering operation of robots by learning. J. Robot. Syst. 1984, 1, 123-140. [CrossRef]

13. Li, Y.; Jiang, W. Fractional order non linear systems with delay in iterative learning control. Appl. Math. Comput. 2015, 257, 546-552.

14. Zhu, Q.; Hu, G.D.; Liu, W.Q. Iterative learning control design method for linear discrete-time uncertain systems with iteratively periodic factors. ET Control Theory Appl. 2015, 9, 2305-2311. [CrossRef]

15. Li, X.D.; Chow, T.W.; Ho, J.K. 2D system theory based iterative learning control for linear continuous systems with time delays. IEEE Trans. Circuits Syst. Regul. Pap. 2005, 52, 1421-1430.

16. Hamidaoui, M.; Shao, C.; Haouassi, S. A PD-type Iterative Learning Control Algorithm for One-dimension Linear Wave Equation. Int. J. Control Autom. Syst. 2020, 8, 1045-1052. [CrossRef]

17. Huang, D.; Li, X.; He, W.; Zhang, S. Iterative learning control for boundary tracking of uncertain nonlinear wave equations. J. Frankl. Inst. 2018, 355, 8441-8461. [CrossRef]

18. Dai, X.; Mei, S.G.; Tian, S.P.; Yu, L. D-type iterative learning control for a class of parabolic partial difference systems. Trans. Inst. Meas. Control 2018, 14, 3105-3114. [CrossRef]

19. Dai, X.; Tu, X.; Zhao, Y.; Tan, G.; Zhou, X. Iterative learning control for MIMO parabolic partial difference systems with time delay. Adv. Differ. Equ. 2018, 18, 344. [CrossRef]

20. Zhengjie, L. Iterative Learning Control Algorithm for Optimal Path of Robot industrial manipulator. J. Intell. Fuzzy Syst. 2021, 40, 5875-5882

21. Yang, X. A PD-Type Iterative Learning Control for a Class of Switched Discrete-Time Systems with Model Uncertainties and External Noises. Discret. Dyn. Nat. Soc. 2015, 2015, 410292 [CrossRef]

22. Zhang, Y.; Liu; L.; Tian, S.; Luo, Y. A Closed-loop PD-type Iterative Learning Algorithm for Discrete Singular Systems. In Proceedings of the 28th Chinese Control and Decision Conference (CCDC), Yinchuan, China, 28-30 May 2016; Volume 40, pp. 4262-4265.

23. Yovchev, K.; Delchev, K.; Krastev, E. Advances in Robot Design and Intelligent Control. In Computer Simulation of Bounded Error Algorithm for Iterative Learning Control; Rodić, A., Borangiu, T., Eds.; Springer International Publishing: Cham, Switzerland, 2017. 